\title{
Heilige Institutionen? - Regelungen von Nutzungsansprüchen an Ressourcen von Nationalparks
}

\section{Norman Backhaus, Michael Kollmair, Zürich}

\section{Einleitung}

An Nationalparks werden unterschiedlichste Nutzungsansprüche gestellt. Sie bergen eine Vielzahl von Ressourcen, deren Nutzung von verschiedenen Akteuren getätigt, angestrebt, propagiert, untersagt oder angefochten wird. Die Ansprüche sind oftmals unvereinbar, doch aus dem jeweiligen Blickwinkel der beteiligten Akteure berechtigt - eine Einsicht, die sich erst im Zuge des partizipativen Schutzgebietsmanagements durchgesetzt hat (BORRINI-FEYERABEND 1997). Weder kann eine neutrale Instanz, welche die objektive Berechtigung verschiedene Ansprüche beurteilen könnte, ausgemacht werden (vgl. ElKanA 1986, LuHMANN 1988), noch stehen sich in der Praxis verschiedene Ansprüche in der «Arena Nationalpark» gleichberechtigt gegenüber.

Entsprechend der Machtausstattung derjenigen, die Ansprüche stellen, können diese besser oder schlechter durchgesetzt werden. In Bezug auf bestehende Nationalparks kann der Schutzgedanke als dominanter Anspruch bezeichnet werden. Dieser wird zu einem großen Teil auch akzeptiert, doch gibt es Stimmen - in der Regel von weniger mächtigen Gruppen -, die die Schutzziele von Nationalparks ablehnen (vgl. Colchester 1997). Der Naturschutz ist also nur eine Ebene der «Arena Nationalpark». Die verschiedenen Ansprüche sind Ergebnis von Überlegungen, Entscheidungen und Handlungen. Diese sind ihrerseits in Strukturen der Alltagspraxis eingebunden, welche sich durch veränderte Rahmenbedingungen - wiederum Ergebnis von Handlungen - verändern können (vgl. "Die Theorie der Strukturierung" von ANTHONY GIDDENS 1995). Die Rahmenbedingungen bzw. Regeln, an die sich Handelnde halten - vor allem wenn sie längerfristigen Bestand haben -, können auch als Institutionen bezeichnet werden (vgl. NORTH 1990). Im Folgenden werden somit unter Institutionen handlungsrelevante Regelungen verstanden und nicht Körperschaften im Sinne von Organisationen, wie dies v.a. im Alltag gebräuchlich ist. Bezüglich der Handlungsarena Nationalpark gehen wir der Frage nach, inwiefern die internationale Institution der Unterschutzstellung andere Regelungen - wie z.B. die Weideoder Jagderlaubnis oder die touristische Nutzung unterdrücken, tolerieren, ermöglichen oder durchsetzen. Wir konzentrieren uns dabei auf Schutzgebiete der IUCN-Kategorie II (vgl. Kap. 3.2), die 30\% der weltweit geschützten Fläche von 13,2 Mio. km² ausmachen und die zumeist auch «Nationalparks» genannt werden (IUCN 1994).

Im Folgenden wollen wir zunächst der Frage nachgehen, welche Ansprüche überhaupt an Nationalparks gestellt werden, bzw. welche Verfügungsrechte im Sinne des «Environmental Entitlements»-Ansatzes (Leach, Mearns \& Scoones 1999) - an den in ihnen wahrgenommenen Ressourcen bestehen, wie sie sich durchsetzen können und welche Institutionen dabei eine Rolle spielen. In der «Handlungsarena Nationalpark» laufen nahezu idealtypisch Prozesse ab (z.B. Partizipation der Lokalbevölkerung am Tourismus oder am Zugang zu natürlichen Ressourcen eines Parks), die durch ein breites Spektrum institutioneller Regelungen beeinflusst werden, die zwischen lokaler und internationaler Ebene angesiedelt sind. Die Interaktionen zwischen Gesellschaft und natürlicher Umwelt müssen dabei dynamisch betrachtet werden, da sie weder in Vergangenheit noch in der Zukunft uniform oder harmonisch waren oder sein werden. Unser Ziel ist es zum einen, die verschiedenen Nutzungsansprüche, die an Nationalparks generell gestellt werden, und die damit verbundenen Probleme aufzuzeigen. Zum anderen wollen wir anhand zweier Fallbeispiele - Tourismus im Gunung Mulu Nationalpark in Ostmalaysia (die Feldarbeiten wurden unterstützt durch die Kommission für Reisestipendien der Schweizerischen Akademie der Naturwissenschaften) und Weidenutzung im Khaptad Nationalpark in Westnepal - die Interdependenzen von Ansprüchen und der Institution Naturschutz diskutieren und damit einen Beitrag zum Erklärungspotenzial des Environmental EntitlementsAnsatzes leisten.

\section{Der Environmental Entitlements-Ansatz}

Erklärt man einen Ausschnitt der Erdoberfläche zum Naturschutzgebiet, so hat dies meist eine Veränderung der Zugangs- und Nutzungsmöglichkeiten im betreffenden Gebiet zur Folge. Dadurch eröffnen sich bestimmten Akteuren Optionen für Handlungen, andere verschließen sich jedoch. Es werden somit auch

Im Text gelten personenbezogene Bezeichnungen als geschlechtsneutral. 
unterschiedliche Ansprüche - neue und alte - an Zugangs- und Nutzungsmöglichkeiten des unter Schutz gestellten Gebietes gestellt. Doch nicht alle, die einen Anspruch stellen, haben auch formal einen einlösbaren Anspruch. Mit dem «Environmental EntitlementsAnsatz» (LEACH et al. 1999) wird versucht, dieses Problem analytisch zu fassen. Basierend auf einem erweiterten verfügungsrechtlichen Ansatz (SEN 1981), bezieht er sich aber auch auf die "New Ecology», die «New Institutional Economics», die "Theorie der Strukturierung» und auf die «historische Landschaftsgenese». Er wird herangezogen, um die Zugänge unter-

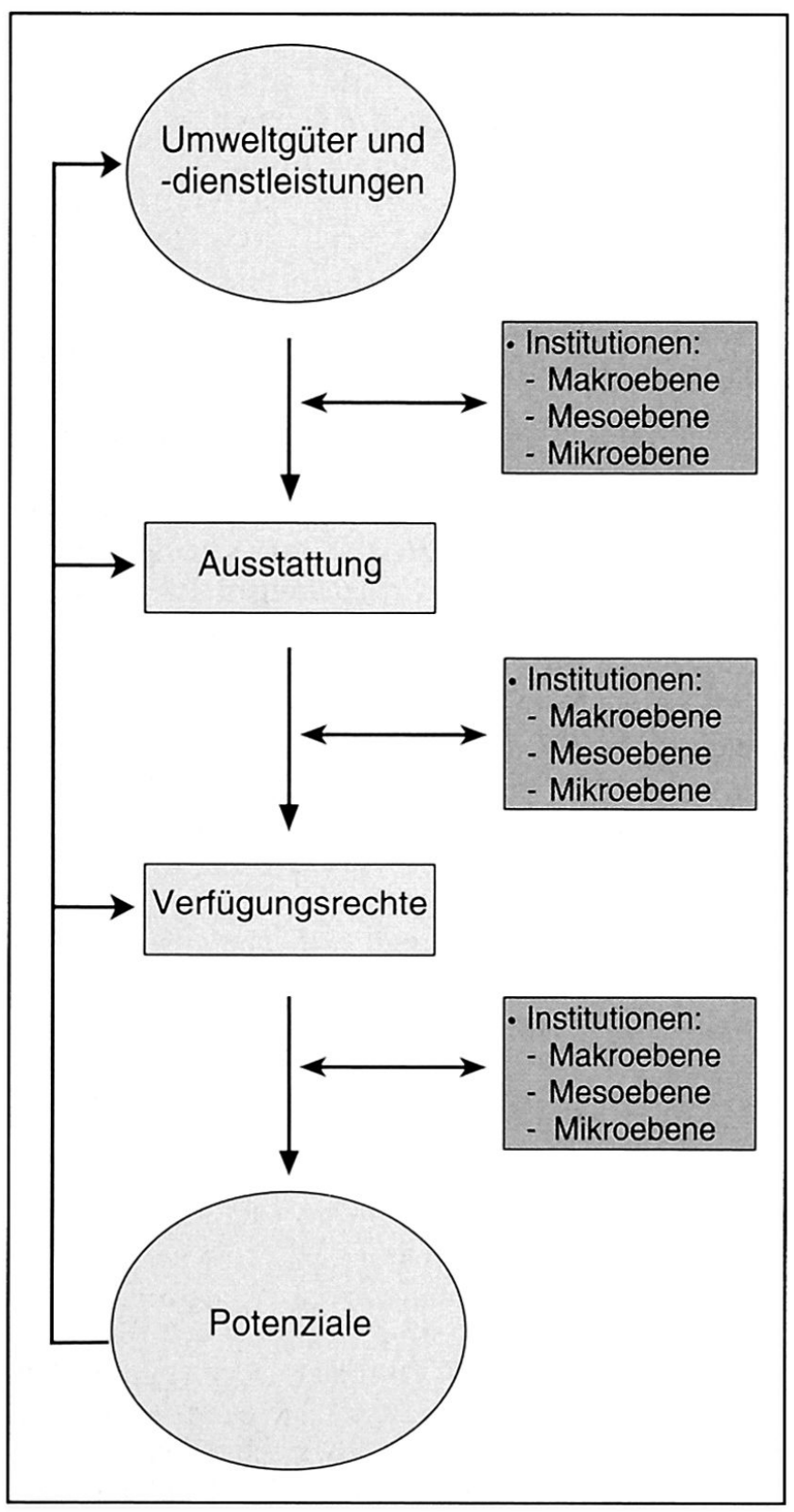

Abb. 1: Der verfügungsrechtliche Ansatz The environmental entitlement framework Schéma des droits de disposition schiedlicher sozialer Akteure zu Umweltgütern zu analysieren. Zentrale Bedeutung wird bei diesem Prozess den Institutionen als Mediatoren der Mensch-UmweltInteraktion zugemessen, wobei die Umsetzung der Ausstattungen dieser Akteure (Endowments) mittels ihrer Verfügungsrechte (Entitlements) zu Potenzialen (Capabilities) im Mittelpunkt steht (vgl. Abb. 1). Institutionen können als Regeln verstanden werden, nach denen sich menschliches Handeln richtet (NORTH 1990: 3 ) und die wiederum durch Handlungen reproduziert werden (Giddens 1995: 69). Für die Analyse verschiedener Interessen beim Naturschutz ist die Betrachtung der daran beteiligten Institutionen hilfreich, da so deutlich wird, dass verschiedene Akteursgruppen sich in unterschiedliche Institutionen eingebunden sehen und dementsprechend bei ihren Handlungen auf unterschiedliche Regeln zurückgreifen. Doch können wir feststellen, dass es einerseits - analog zu den Ansprüchen - mächtigere und weniger mächtige Institutionen und anderseits mehr und weniger verbreitete Institutionen gibt. Ziel ist die Analyse der Zusammenhänge zwischen ökologischer und sozialer Dynamik und der Nutzung natürlicher Ressourcen durch bestimmte soziale Gruppen. Die Ergebnisse, die dadurch gewonnen werden, können dazu dienen, externe Interventionen gezielter zu steuern, z.B. um die Verfügungsrechte einer bestimmten sozialen Gruppe zu schützen oder den Output einer natürlichen Ressource besser nutzen zu können.

\section{Werte und Institutionen in Naturschutzgebieten}

Werte, die in Umweltgütern gesehen werden, bestehen nicht per se, vielmehr sind sie an Vorstellungen und Wünsche von Individuen und Gruppen gebunden und damit auch Veränderungen unterworfen. Sie sind gekoppelt an Bedürfnisse und Interessen von Akteuren und hängen auch von kulturellen Vorstellungen ab.

\subsection{Werte}

Heute wird die Unterschutzstellung eines Gebietes vor allem mit der hohen Biodiversität oder mit der Einzigartigkeit eines Ökosystems begründet. Die Artenvielfalt wird von internationalen Naturschutzorganisationen als Wert an sich betrachtet, sie wird als Indikator für Intaktheit, Unberührtheit und Integrität eines Gebietes erachtet. Vor allem in westlichen Gesellschaften ist der Gedanke, «ursprüngliche» Natur zu schützen, stark verankert. Wo zunächst Erbauung und der Schutz bestimmter Spezies im Vordergrund standen, ist heute die globale Bedeutung von Biodiversitäts- und Ökosystemschutz in den Vordergrund gerückt worden. Die Vielfalt von Arten und Lebensräumen wird als wichtiger Faktor für das Überleben der Menschheit, ja des Planeten gewertet (vgl. WWF 2000). 
Über die Werte, die mit der Biodiversität verbunden sind, wird meist in sehr abstrakter und generalisierter Weise diskutiert. Dabei wird zwischen der Erhaltung der Biodiversität und dem Wohlergehen der Menschheit - zumindest für lange Zeiträume und die gesamte Erde - ein direkter Zusammenhang postuliert (WILSON \& Peter 1988). Es scheint uns daher sinnvoll, hier die wichtigsten Werte, die man der Biodiversität und Ökosystemen zuschreiben kann, zu identifizieren und deren Relevanz für verschiedene Akteursgruppen aufzuzeigen. Eine umfassende Darstellung scheint (zumindest an dieser Stelle) nicht möglich zu sein, da diese Werte im lokalen und regionalen Kontext sehr vielfältig sind und stark differieren können. In Abwandlung einer Systematisierung von BlaikIE \& JeAnRENAUD (1997) sollen sie in «direkte», «indirekte» und «optionale» Nutzwerte unterteilt werden. Wie diese Werte monetär umgesetzt werden können (vgl. KüPFER 2000) wird hier nicht weiter erläutert. Die Perzeption und Gewichtung von Werten kann von den im Naturschutz beteiligten Akteursgruppen recht verschieden sein.

Direkte Nutzwerte: Die direkten Nutzwerte an einem Schutzgebiet sind die offensichtlichsten. Meist werden diese Werte von der lokalen Bevölkerung, aber auch von den touristischen Nutzern eines Schutzgebietes hervorgehoben. Für die Lokalbevölkerung kann eine große Biodiversität häufig Grundlage für die Bestreitung ihres Lebensunterhalts sein. So ernten die Penan in Mulu Sagopalmen und jagen Affen und Wildschweine. Im Khaptad Nationalpark werden die Waldund Weideflächen intensiv genutzt und eine Vielfalt von Medizinalpflanzen (illegal) gesammelt. Weniger an der Vielfalt, als an spezifischen Spezies sind, neben der Lokalbevölkerung, zum Beispiel Holzkonzerne, aber auch (Trophäen-)Jäger und Tierfänger interessiert. Schutzgebiete - allen voran tropische Primärwälder - beinhalten oft alte Baumbestände, die so an anderen Orten kaum mehr anzutreffen sind. Jäger und Tierfänger sind an Tierarten oder deren Produkten interessiert, die sie gewinnbringend an vor allem ausländische Kundschaft verkaufen können.

Laut IUCN-Klassifizierung sollen über $80 \%$ aller Schutzgebiete (Kategorie II bis VI) auch einer Erholungs- oder Erbauungsfunktion dienen (IUCN 1994, IUCN 1998) - eine Funktion, die man als einen direkten Nutzwert bezeichnen muss. Das Gefühl, sich in einer ursprünglichen, authentischen, unverbauten und unverschmutzten Naturlandschaft aufzuhalten und zu bewegen, wird von vielen Parkbesuchern als Wert an sich betrachtet. An den ästhetischen Wert - der oft mit Exotik und Abenteuer verbunden wird - eines Schutzgebietes gekoppelt wird oft auch ein erzieherischer, bei dem ökologische Zusammenhänge aufgezeigt und in der Bevölkerung verbreitet werden sollen.

Die Tourismusindustrie wirbt mit der Biodiversität für Nationalparks und bedient dabei die Ansprüche von
Touristen, die einerseits eine ursprüngliche und intakte Natur erleben möchten und die anderseits hoffen, viele dieser Arten auch in ihrer authentischen Umgebung sehen und hören zu können. Authentizität wird hier als sozial konstruiert und nicht als etwas objektiv Bestehendes verstanden (vgl. BackHaus 2000). Die Möglichkeit ein Panzernashorn oder einen Tiger in «freier Wildbahn» zu sehen, lockt über 80'000 Touristen jährlich in den Chitawan Nationalpark in Nepal (MüLLER-BöKER 2000). Doch auch die überwältigende biologische Vielfalt bestimmter Gebiete kann zur Werbung dienen: so wird der Taman Nationalpark in Malaysia mit seinen 350 Singvögelarten, der Kinabalu Park auf Borneo mit über 1200 Orchideenarten angepriesen (vgl. u.a. CubitT \& PAYNe 1996). Aber auch der mit einem Schutzgebiet verbundene Wert «landschaftlicher Schönheit» stellt einen direkten Nutzwert dar. Naheliegend ist es, diesen Wert mit Tourismus zu koppeln. Die ästhetischen Werte, die die lokale Bevölkerung mit einem Gebiet verbindet, werden dagegen häufig übersehen (MÜLlER-BÖKER \& KOlLMAIR 2000).

Neben an physisch-materielle Gegebenheiten gebundenen direkten Nutzwerten kann ein Nationalpark auch immaterielle Werte bergen. Als klassisches Beispiel gelten heilige Berge (Bernbaum 1996) und an spezifische Lokalitäten gebundene Erscheinungen. So stellten die obersten 1000 Meter des als heilig betrachteten Mt. Kinabalu im gleichnamigen Park für die Idaan-Dusun-Kadazan eine Tabuzone dar, die nur von den Toten betreten werden durfte (HARRISSON 1996:3) Der Khaptad Nationalpark in Nepal wurde auf Initiative eines dort ansässigen hinduistischen Heiligen ausgewiesen. Im Zentrum des Parks steht eine $5 \mathrm{~km}^{2}$ große «heilige Zone» in der strikte Nutzungsbeschränkungen gelten (Kollmair \& Müller-BöKer 2001). Aber auch einzelne Spezies können bestimmten Gruppen heilig sein. So gelten Nashornvögel bei vielen Völkern in Sarawak als Verkünder von Botschaften und genießen Schutz vor Verfolgung (Freeman in Thambiah 2000).

Indirekte Nutzwerte: Als indirekte Nutzwerte werden die Werte bezeichnet, die einer Gemeinschaft gesamthaft zugute kommen. Auf diese Werte berufen sich vor allem internationale Naturschutzorganisationen und nationale Interessenvertreter. Typische Beispiele für diese Kategorie sind der Schutz vor Erosion und des Wasserhaushaltes. Gerade in tropischen Regionen werden Wälder aus Wasserschutzgründen vor dem Einschlag geschützt, da sie einen grossen Teil der Starkregen absorbieren und einen schnellen, erosiven Abfluss verhindern. Es besteht diesbezüglich auch ein öffentliches Interesse der Regionalbevölkerung am Erhalt von Waldzonen. An der Quantität von Biomasse bzw. an Pflanzen, die $\mathrm{CO}_{2}$ abbauen können, sind Kreise interessiert, die den weltweiten Anstieg des Treibhausgases bremsen wollen. 
Optionale Nutzwerte: Unter optionalen Nutzwerten fasst man alle potentiellen zukünftigen direkten und indirekten Nutzungen zusammen, deren Option man sich durch die Unterschutzstellung erhalten kann. Im Moment ist es vor allem die genetische Biodiversität, die zunehmend für die Pharmaindustrie und Biotechnologie interessant wird, die hier einen Fundus von möglichen Wirkstoffen und genetischen Ressourcen sieht, die es noch zu entdecken gilt (QUEROL 1992).

\subsection{Internationale Naturschutzregeln als «heilige Institutionen»}

Bei der Ausweisung und Abgrenzung von Schutzgebieten spielen internationale Naturschutzinstitutionen die entscheidende Rolle. Sie bestimmen, welche Kriterien ein Schutzgebiet erfüllen muss, welche Nutzungen erlaubt oder erwünscht sind und über welche «Qualitäten» es verfügen muss. Manifestiert haben sich diese Institutionen in internationalen Naturschutzorganisationen wie IUCN und WWF, aber auch der UNESCO, die heute die wichtigsten internationalen Akteursgruppen im Bereich Naturschutz sind.

Wie bereits erwähnt, haben diese Organisationen ihre Programme (und damit die wirksamen Institutionen) immer wieder revidieren müssen. Während es früher Ziel des Naturschutzes war, die «ursprüngliche Natur» durch den Ausschluss physischer Nutzung zur Erbauung der Menschen zu erhalten (das sogenannte Yellowstone-Modell), ist nun das Management von Schutzgebieten - oft mit menschlichen Eingriffen, die den Erhalt der Biodiversität gewährleisten sollen - erwünscht (Ghimire \& Pimbert 1997). Die aktuellen «Dogmen» lauten: «Naturschutz und Entwicklung sind nur gemeinsam möglich» und «der Schutz der Biodiversität ermöglicht uns das Überleben» (vgl. IUCN, UNEP \& WWF 1991: 1).

Diese beiden Aussagen belegen eindrücklich den universellen Anspruch, den die Institution Naturschutz gegenüber allen anderen Nutzungszielen (auch auf lokaler Ebene) stellt. Der Naturschutz wird somit zu einer unantastbaren «heiligen Institution», zum mächtigsten Anspuch, der nicht in Frage gestellt werden darf. Auf die Frage, ob diese allgemeinen, auf internationaler Ebene sicherlich anerkannten Ziele, auch auf lokaler Ebene gelten, soll später eingegangen werden. Eine Besonderheit der Arena Nationalpark gegenüber Nutzungskonflikten in anderen Bereichen ist folglich darin zu sehen, dass ein einmal ausgewiesenes Schutzgebiet (zumindest in absehbarer Zukunft) nicht mehr rückgängig gemacht werden kann, das primäre Ziel «Naturschutz» bleibt so - auch bei sich wandelnden Nutzungsansprüchen - dem Gebiet erhalten.

Für die in der Kategorie II ausgewiesenen Parks gelten folgende übergeordnete Regelungen, nach denen sie geführt werden sollen (IUCN 1994:19):
- Schutz natürlicher Regionen und landschaftlich reizvoller Gebiete von nationaler und internationaler Bedeutung für geistige, wissenschaftliche, erzieherische, touristische oder Erholungszwecke.

- Dauerhafter Erhalt charakteristischer Beispiele physiographischer Regionen, Lebensgemeinschaften, genetischer Ressourcen und von Arten in einem möglichst natürlichen Zustand, damit ökologische Stabilität und Vielfalt gewährleistet sind.

- Besucherlenkung für geistig-seelische, erzieherische, kulturelle und Erholungszwecke in der Form, dass das Gebiet in einem natürlichen oder naturnahen Zustand erhalten wird.

- Beendigung und Unterbindung von Nutzungen oder Inanspruchnahme, die dem Zweck der Grundlage für die Ausweisung entgegenstehen.

- Respektierung der ökologischen, geomorphologischen, religiösen oder ästhetischen Attribute, die Grundlage für die Ausweisung waren.

- Berücksichtigung der Bedürfnisse der lokalen Bevölkerung einschließlich deren Nutzung bestehender Ressourcen zur Deckung ihres Lebensbedarfes unter der Bedingung, dass diese keinerlei nachteilige Auswirkungen auf die anderen Managementziele haben.

Im Folgenden werden die spezifischen Wertvorstellungen zugrunde liegenden institutionellen Regelungen anhand zweier Beispiele - Gunung Mulu und Khaptad - dargelegt.

\section{Die touristische Nutzung des Gunung Mulu Nationalparks in Malaysia}

Gunung Mulu Park (kurz Mulu), mit $528 \mathrm{~km}^{2}$ der grösste Nationalpark im malaysischen Gliedstaat Sarawak auf Borneo, wurde 1974 gegründet und 2000 in die Liste der World Heritage Sites der UNESCO aufgenommen (UNESCO 2000). Mulu grenzt an Brunei Darussalam und ist von der Küstenstadt Miri mit kleinen Propellerflugzeugen innert 45 Minuten und mit Flussbooten innert Tagesfrist erreichbar (vgl. Abb. 2). Seine touristischen Hauptattraktionen sind Höhlenkammern, Karstformationen, Gipfel sowie ein tropischer Regenwald mit einer großen Artenvielfalt. Vor der Ausweisung als Schutzgebiet war die Region kaum besiedelt. Einzig nomadisierende Penan nutzten den heutigen Nationalpark als Lebensraum. Vor allem während der 80er Jahre wanderten Berawan, Kenyuah und Kelabit flussaufwärts und besiedelten ein Gebiet nahe des Hauptquartiers und Parkeingangs, u.a. in der Hoffnung auf Einkommen aus dem Tourismusgeschäft. Ein Großteil der etwa 300 Penan des Mulu-Gebietes ist heute sesshaft und lebt in zwei Dörfern am Rande des Parks.

Im Folgenden wollen wir Institutionen, die bei der touristischen Nutzung des Gunung Mulu Parks eine 


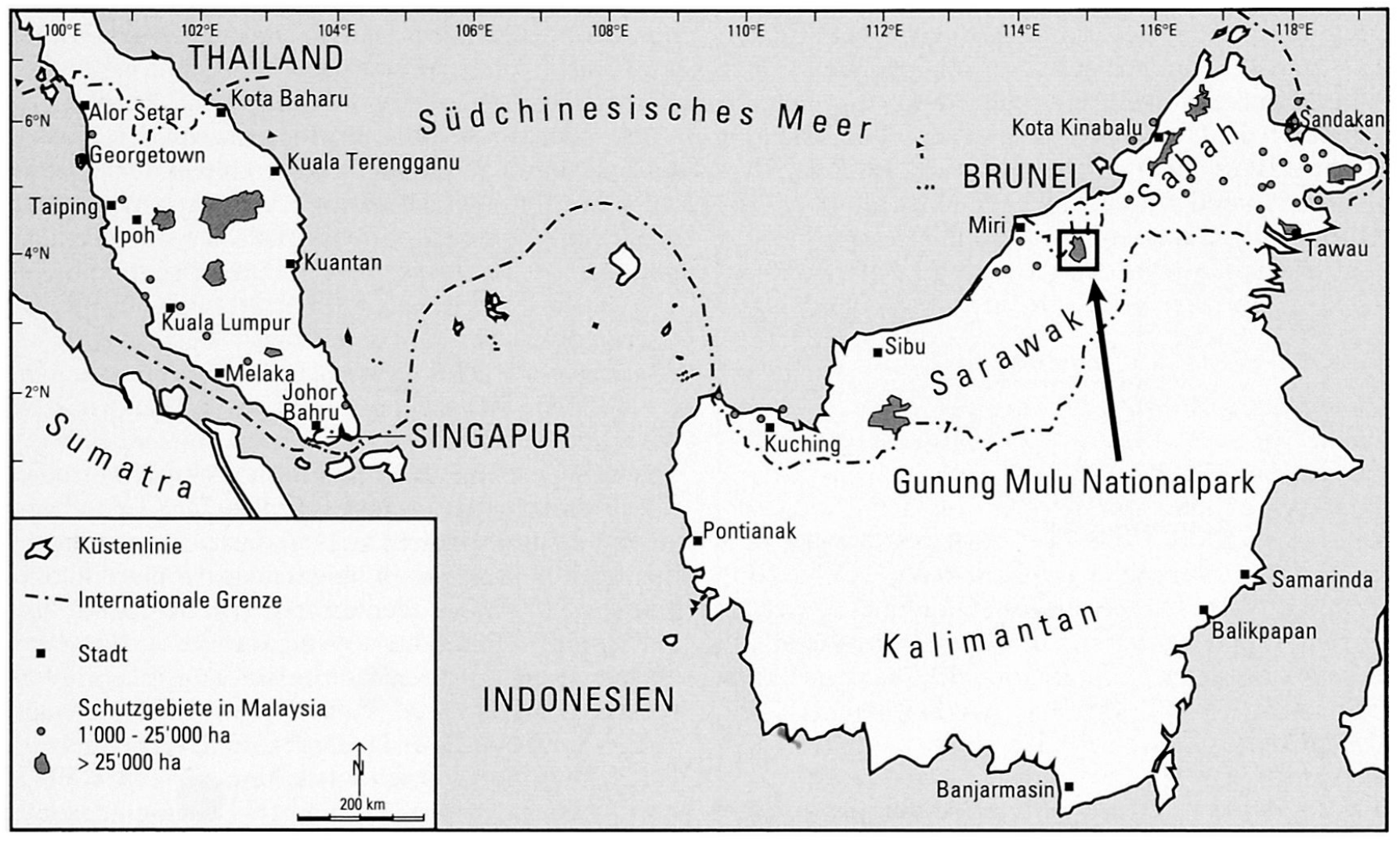

Abb. 2: Lage des Gunung Mulu Nationalparks in Malaysia Location of Gunung Mulu National Park in Malaysia Localisation du parc national de Gunung Mulu en Malaisie Entwurf: N. BACKHAUS

Rolle spielen, herausgreifen und anhand des Environment Entitlements-Ansatzes beschreiben und analysieren (vgl. Abb. 3).

\subsection{Werte und Ressourcen in Mulu}

Touristisch bedeutsam ist Mulu mehrheitlich aus den gleichen Gründen, aus denen das Gebiet unter Schutz gestellt wurde: eine hohe Biodiversität, spektakuläre Karstformen mit dem höhlenreichsten Berg der Welt, dem Gunung (malaiisch Berg) Mulu im Zentrum, 17 Vegetationszonen sowie zwei Millionen Fledermäuse, die (fast) allabendlich aus dem weltgrößten Höhleneingang herausfliegen, um bei ihrer nächtlichen Nahrungssuche mehrere Tonnen von Insekten zu vertilgen.

Warum diese Eigenschaften für viele potentielle Touristen Werte darstellen, hängt von vielen Gründen ab. Ein wichtiger Faktor ist die in modernen Gesellschaften vollzogene Organisation von Arbeit und Freizeit als separate - jedoch nicht unabhängige - Sphären (URRY 1990). In der Freizeit, speziell im Urlaub, suchen Touristen in der Regel Dinge und Orte auf, die nicht direkt mit ihrer Arbeit zu tun haben. Bei der Auswahl ihrer Ziele helfen ihnen Informationen - vor allem visuelle Bilder -, zu denen sie in ihrem Alltag Zugang haben. Die Fülle von Bildern exotischer Orte, die ihnen durch verschiedenste Kanäle ins Haus gebracht wird, lässt den Wunsch reifen, das Reich der Farben, des Spektakels, der Größe und Exotik zu betreten und den Käfig der grauen Routine zu verlassen (BAUMAN 1997). Im modernen Alltag, der häufig als «künstlich» wahrgenommen wird, vermissen sie die Authentizität, die ihnen beispielsweise über Berichte und Bilder zur einzigartigen Bedeutung von Regenwäldern oder «ursprünglichen» Landschaften näher gebracht wird. Dabei hat die Unterschutzstellung eines Gebietes die Funktion einer Garantie für diese Authentizität. Einzigartige Landschaften und große Artenvielfalt werden somit zu Zeichen für eine größtmögliche Authentizität, die so zum eigentlichen Wert für viele potentielle Reisende wird (vgl. BACKHAUS 2000).

\subsection{Endowments: Wer hat das Recht, Mulu zu besuchen?}

Grundsätzlich haben alle Menschen die Möglichkeit, Mulu zu besuchen, denn die Regeln des Parks sehen 


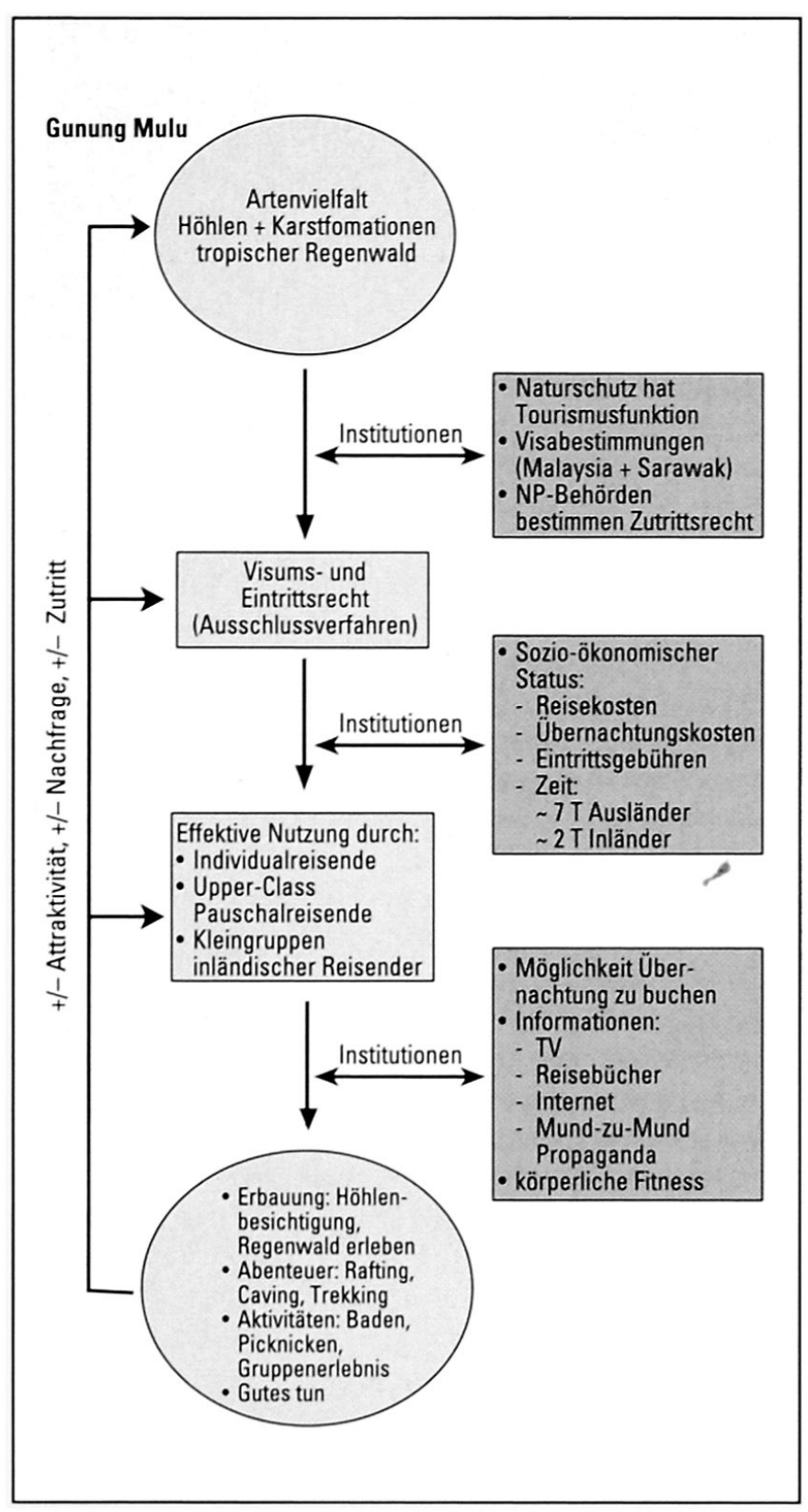

Abb. 3: Der verfügungsrechtliche Ansatz: Beispiel Tourismus im Gunung Mulu Nationalpark in Malaysia The environmental entitlement framework: tourism in the Gunung Mulu National Park in Malaysia Schéma des droits de disposition: l'exemple du tourisme dans le parc national de Gunung Mulu en Malaisie

eine touristische Nutzung explizit vor (vgl. IUCN 1994), mit der auch geworben und für die Infrastruktur bereitgestellt wird (vgl. HutTon 1998, SarawaK TourISM BOARD 1997). Damit wird das Schutzgebiet besser zugänglich als zum Beispiel das Umland, das ähnliche Eigenschaften aufweist. Das Wissen darüber, dass man Nationalparks in der Regel besuchen darf und dass die nötige Infrastruktur für einen Besuch vorhanden ist, ist weit verbreitet. Bei anderen - möglicherweise ebenso attraktiven Gebieten - ist dies nicht der Fall. Dass Mulu nicht von Reisenden überschwemmt wird - was ökologisch problematisch wäre und seine Attraktivität wieder senken würde -, liegt an verschiedenen institutionellen Regelungen. Bislang gibt es noch keine zahlenmäßige Beschränkung der Besucher, da die Tragfähigkeit des Parks noch nicht als überschritten gilt. In der Hochsaison - den europäischen Sommerferien, die teilweise mit malaysischen Schulferien überlappen - im Juli und August werden pro Tag 30 bis 60 Neuankünfte registriert. Bei einer durchschnittlichen Verweildauer von zwei Nächten bzw. drei Tagen ergibt dies eine tägliche Spitzenbelastung von höchstens 200 Besuchern, die sich auf verschiedene Attraktionen im Park aufteilen. Diese Zahl wird (noch) nicht als bedenklich oder schädigend angesehen, allerdings sprechen verschiedene Ranger bereits davon, dass sich die Fledermäuse von den Besuchern der Höhle gestört fühlen. Wenn auch keine formellen Zugangsbeschränkungen bestehen, so gibt es informelle: die Kapazität der Flugzeuge, die Mulu anfliegen sowie die Unregelmässigkeit der Bootsverbindungen. Eine Twin-Otter-Propellermaschine - größere Flugzeuge können (noch) nicht in Mulu landen - fasst 19 Passagiere, und es werden höchstens sechs Flüge pro Tag geflogen. Für viele Reisende, die etwas mehr Zeit zur Verfügung haben, wäre die Reise mit dem Boot auf dem Fluss zwar attraktiv, doch fahren die Boote unregelmäflig, und die Anschlüsse sind nicht immer gewährleistet. Die limitierenden Verkehrsverbindungen stellen somit eine effektive Einschränkung des Zugangs dar.

\subsection{Entitlements: Wer besucht Mulu?}

Die Regelung, dass der Park prinzipiell allen zugänglich ist, bedeutet jedoch nicht, dass er damit allen Interessierten ohne Einschränkungen frei zugänglich wäre und Touristen darin tun und lassen könnten, was sie wollen. Die Art und Weise, wie sich Touristen den Park aneignen können, wird über verschiedene institutionelle Regelungen organisiert. Eine offensichtliche Regelung ist die Verfügbarkeit von Geld, um überhaupt nach Sarawak und Mulu reisen zu können. Praktisch alle Besucher kommen aus Übersee auch das Gros der malaysischen - und müssen sich Flüge und Übernachtungen leisten können. Dies schränkt die Zahl der potentiellen Reisenden stark ein. In Mulu selbst ist nicht die Zahl der Übernachtungsmöglichkeiten ein limiterender Faktor der Flaschenhals liegt wie gesagt beim Transport - sondern die Verfügbarkeit von geeigneten Unterkünften. Die Parkadministration selbst bietet günstige Betten an, doch sind diese schnell ausgebucht. Daneben gibt es außerhalb des Parks zwei günstige «Homestays» und ein Viersternehotel. Die Struktur des Übernachtungsangebots führt zu einer Segmentierung der Reisenden (1999 waren es insgesamt 12'000) in 

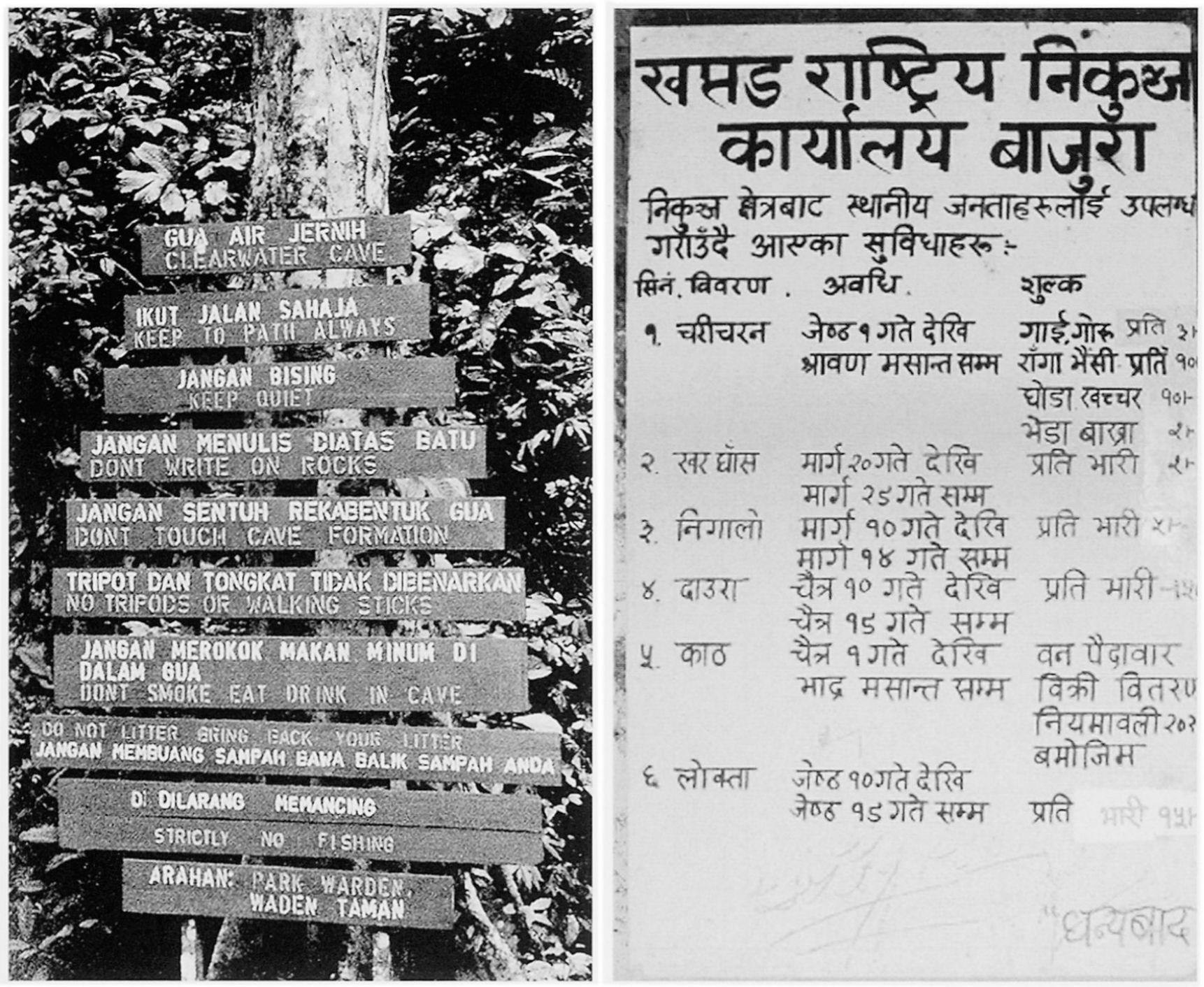

Abb. 4: Bekanntmachung von Nutzungsregelungen im Gunung Mulu und Khaptad Nationalpark

Regulation boards in the Gunung Mulu and Khaptad National Parks

Affichage des régimes d'utilisation dans les parcs nationaux de Gunung Mulu et de Khaptad

Photos: N. Backhaus/M. Kollmair

günstig logierende Individualreisende und Pauschalreisende der gehobenen Preisklasse - die Schicht, die Arrangements der Mittelklasse nachfragt, fehlt.

Nachdem man sich im Park registriert und die Eintrittsgebühr von 5 Ringgit (etwas mehr als 2 $\mathrm{CHF}$ ) bezahlt hat, sind nur bestimmte Zonen und Attraktionen frei zugänglich (vgl. Abb. 4). Auf sogenannten «Plankwalks» können Dipterocarpaceenwald und einige spektakuläre Höhlen durchwandert und besichtigt werden. Die auf den ersten Blick als grober Eingriff in die Natur wahrgenommenen Plankenwege schützen das Ökosystem weit besser als wenn Pfade durch die vielbesuchten Gebiete führen würden, da der Bodenverdichtung sowie dem Abschweifen von Touristen vorgebeugt werden kann. Für weiterge- hendes Eindringen in den Park müssen Reisende einen Führer oder eine Führerin aus den Reihen der Ranger engagieren, wobei es auch hier das in Malaysia allgegenwärtige Motto «take nothing but photographs, leave nothing but footprints» zu berücksichtigen gilt.

\subsection{Capabilities: Was tun Reisende in Mulu?}

Das Gros der Besucher besucht morgens und nachmittags jeweils zwei zugängliche Höhlen (zu Fuss oder mit dem Boot), um dann abends auf das Ausfliegen der Fledermäuse zu warten. Dieses Programm ist in zwei Tagen gut zu bewältigen. Wenige (Individual-)Reisende buchen ein mehrstündiges «Adventure Caving» bei dem schwerer zugängliche Höhlenteile durchstiegen werden und für welches ein minimaler 
Fitnessstandard nicht unter- und eine maximale Körperfülle nicht überschritten werden darf. Auch die zwei- bis dreitägigen Trekkings werden fast ausschließlich von Individualreisenden gemacht. Die verschiedenen Aktivitäten, die Reisende zu ihrer Erholung oder Erbauung betreiben, können sich jedoch auch gegenseitig stören. Vogelbeobachter sehen sich gestört durch Reisende, die den Ausflug in den Park vor allem als Gruppenerlebnis genießen und durch ihren Lärm die Vögel verjagen, Individualreisende betrachten mitunter die Tatsache, dass Pauschalreisende den Park ebenfalls besuchen, bereits als Wertminderung des Erlebnisses. Die Bedürfnisse der Reisenden können also stark variieren, nicht nur zwischen den Individuen, sondern auch tageszeitlich bei derselben Person, die zum Beispiel nach der frühmorgendlichen, stillen Kontemplation am Mittag Lust auf eine rasante Flussfahrt hat (vgl. BackHaus 2000).

\section{Weidewirtschaft im Khaptad Nationalpark in Nepal}

Kontrastierend zur Analyse der stark durch touristische Nutzung geprägten Problemlage in Mulu, wird im Folgenden die Nutzung der natürlichen Ressourcen durch die lokale Bevölkerung untersucht. Am Beispiel der weidewirtschaftlichen Nutzung des Khaptad Nationalparks in Nepal sollen die Endowments, Entitlements und Institutionen veranschaulicht werden, die die Nutzung der natürlichen Ressourcen regeln (vgl. Abb. 5).

Der Khaptad Nationalpark liegt im äussersten Westen Nepals und erstreckt sich in einer Höhenlage von 1600 bis $3300 \mathrm{~m}$ über $225 \mathrm{~km}^{2}$ (vgl. Abb. 6). Bei den angrenzenden Distrikten mit etwa 600'000 Einwohnern handelt es sich selbst im nepalesischen Kontext um eine extrem arme Region (NESAC 1998). Relativ niedrige Produktionsziffern der Landwirtschaft, regelmässige Nahrungsmittelhilfen, aber auch die Absenz von Entwicklungs- und Forschungsprojekten weisen auf die marginale Stellung der Region hin. Temporäre Arbeitsmigration und Emigration sind weit verbreitet.

Der Nationalpark unterscheidet sich schon aufgrund seiner Entstehungsgeschichte grundsätzlich von vielen anderen Parks, denn er wurde auf Initiative eines hinduistischen «Heiligen», dem Khaptad Swami (Baba), gegründet. Dieser in weiten Teilen Nepals bekannte Swami wurde beim König mit dem Wunsch vorstellig, den «heiligen Hain» um seinen Ashram in Tribeni unter Schutz zu stellen. Diesem Wunsch wurde stattgegeben, und innerhalb weniger Monate waren die rechtlichen und administrativen Grundlagen für einen neuen Nationalpark geschaffen.

Obwohl es bislang keinen umfassenden Managementplan für das Schutzgebiet gibt, wurden bereits kurz nach der Ausweisung des Nationalparks im Jahre 1986 Nut-

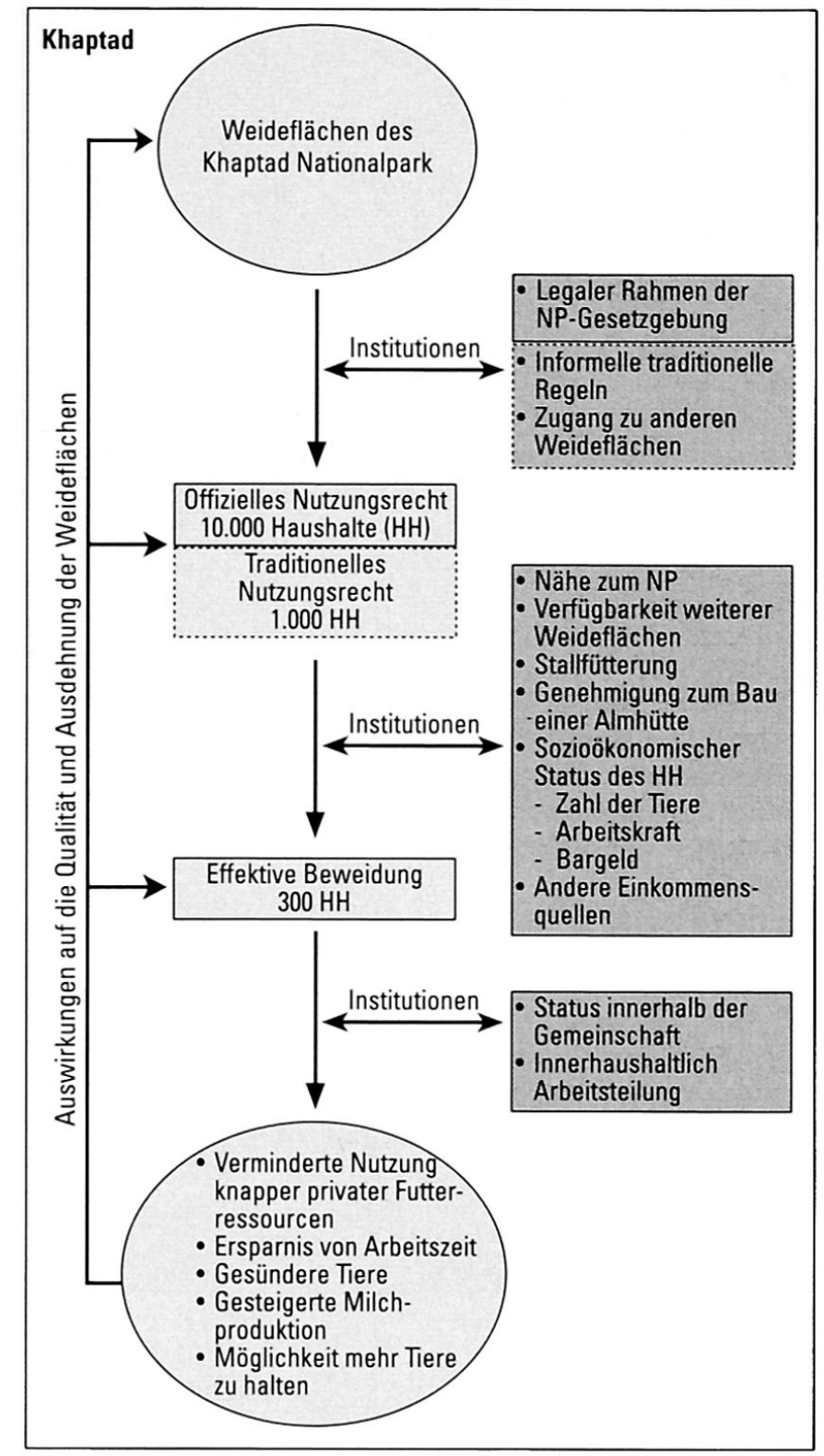

Abb. 5: Der verfügungsrechtliche Ansatz: Beispiel Weidewirtschaft im Khaptad Nationalpark in Nepal The environmental entitlement framework: grazing in the Khaptad National Park in Nepal

Schéma des droits de disposiiotn: l'exemple de l'exploitation des pâturages dans le parc national de Khaptad au Népal

zungsregeln erlassen, die mit einigen Veränderungen im Detail bis heute gültig sind (IUCN 1993). Innerhalb des Nationalparks befinden sich auf einer in $3000 \mathrm{~m}$ gelegenen Hochebene saisonale Sommersiedlungen. Während vier Monaten ist es den Parkanrainern erlaubt, die traditionelle Alpweidewirtschaft fortzuführen und ihre Tiere im Park weiden zu lassen. In stark reglementierter Form (gegen Entgeld und nur für einige Tage im Jahr) dürfen einige weitere Ressourcen des Parks wie Bambus, Seidelbast, Gras 


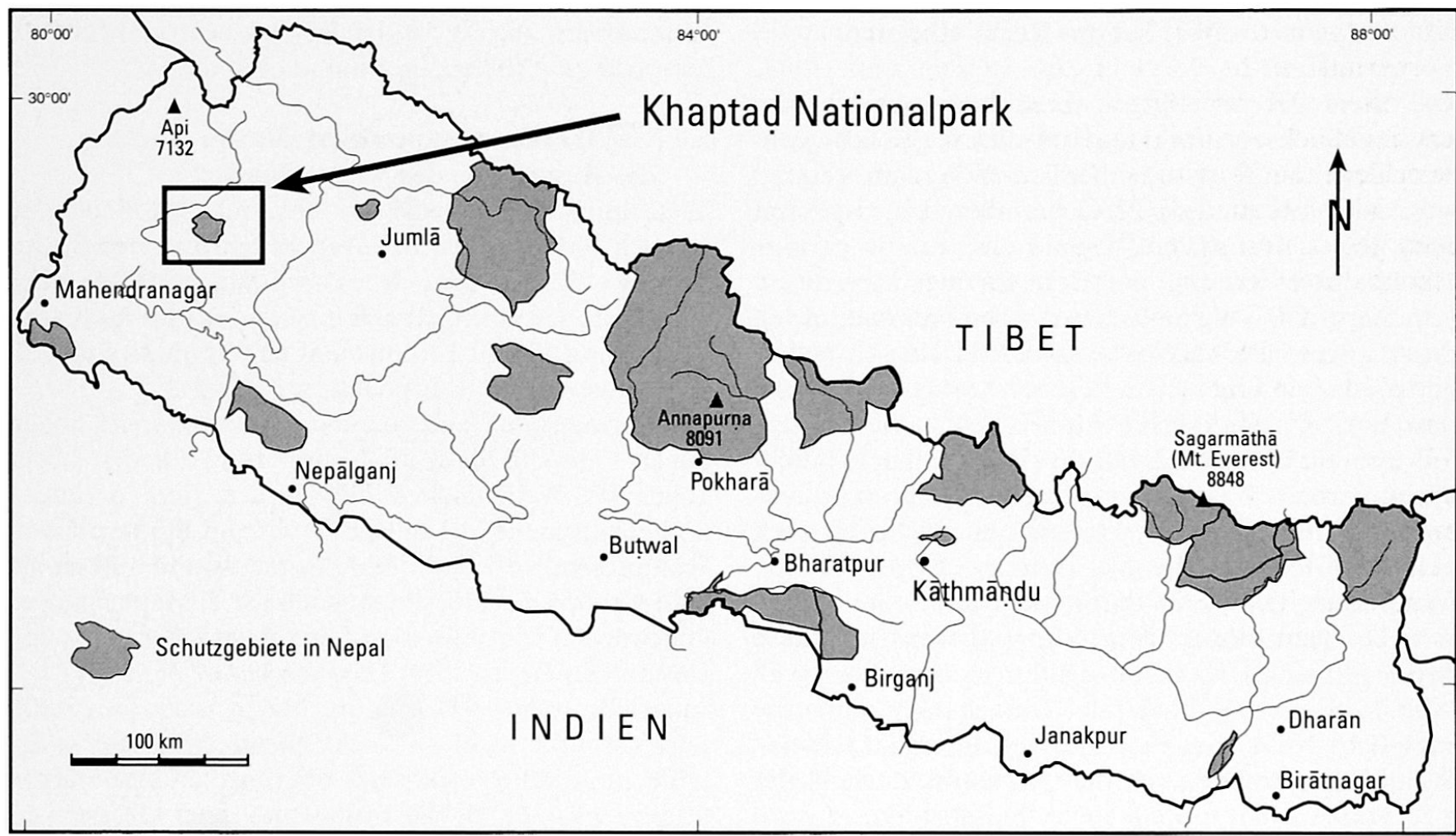

Abb. 6: Lage des Khaptad Nationalparks in Nepal Location of the Khaptad National Park in Nepal Localisation du parc national de Khaptad au Népal Entwurf: M. Kollmair

und Brennholz genutzt werden (vgl. Abb 4). Über die Einhaltung der Nutzungsregeln wachen Personal des zuständigen Ministeriums und eine Einheit der Armee, die im Zentrum des Parks stationiert ist. Westliche Reisende finden selten den Weg nach FarWest-Nepal: In den letzten 15 Jahren wurden insgesamt nur 155 ausländische Besucher und Besucherinnen gezählt (KOLLMAIR \& MÜLLER-BÖKER 2001). Wegen der schlechten verkehrstechnischen Anbindung und der völlig fehlenden touristischen Infrastruktur scheint in naher Zukunft eine intensive Nutzung der Region durch den internationalen Tourismus eher unwahrscheinlich. Aufgrund seiner religiösen Bedeutung werden die im Nationalpark gelegenen heiligen Stätten jedoch von Tausenden einheimischer Besuchern pro Jahr frequentiert.

\subsection{Werte und Ressourcen}

Aus der Sicht des Naturschutzes liegt die Bedeutung des Khaptad Nationalparks darin, dass er als einziger Nationalpark die Ökosysteme des westlichen nepalesischen Hügellands abdeckt. Vor allem wird der sehr gut erhaltene Waldbestand angeführt, aber auch für Nepal seltene See- und Moorökosysteme werden genannt
(IUCN 1993). Zum breiten Faunenspektrum des Parks zählen 223 Vogelarten, die als einzige Tiergruppe relativ gut untersucht ist (INSKIPP 1989). Für die lokale Bevölkerung ist die legale (aber auch illegale) Nutzung der natürlichen Ressourcen zur Aufrechterhaltung ihrer subsistenzorientierten Landwirtschaft von zentraler Bedeutung.

Von sicherlich grösserer Relevanz als die physischen Werte, die dem Park beigemessen werden, sind die kulturellen Werte. Wie bereits erwähnt, ist der Bereich des Khaptad Nationalparks ein religiöses Zentrum von grosser regionaler Bedeutung. Nicht erst seit dem Erscheinen des Khaptad Baba, sondern - laut Auskunft der Bevölkerung - seit vielen Jahrhunderten werden bei Naturheiligtümern und Tempeln auf der Hochfläche religiöse Rituale durchgeführt. Als besonderer Wert wird von der Bevölkerung (und den wenigen Touristen) die stille, friedliche Atmosphäre im Park immer wieder betont. Somit stellt die abwechslungsreiche Landschaft der Hochebene mit dem Wechsel von Weide- und Waldflächen mit Ausblicken auf die schneebedeckten Berge des hohen Himalayas sicherlich den grössten Wert und das entscheidende touristische Potential des Parks dar. 


\subsection{Endowments: Wer hat das Recht, Khaptad zu nutzen?}

Die Weideflächen dürfen ebenso wie die übrigen natürlichen Ressourcen des Parks nur von den Bewohnern einer den Park umgebenden Pufferzone genutzt werden. Diese umfasst 22 Gemeinden mit insgesamt etwa 10'000 Haushalten. Gegen eine relativ geringe Gebühr ist es erlaubt, die Tiere für vier Monate im Sommer auf den Weideflächen der Hochfläche und den direkt angrenzenden Wäldern weiden zu lassen. Befragungen haben ergeben, dass schon vor der Gründung des Parks aber lediglich Personen aus 10 Gemeinden Tiere in den Bereich des jetzigen Nationalparks brachten. Innerhalb dieser zehn Gemeinden waren es aber auch nur Personen aus sehr nah am (jetzigen) Park gelegenen Dörfern, die ihre Tiere auf der Hochfläche sömmerten. Dies lässt darauf schliessen, dass traditionelle Institutionen den Zugang bereits auf diese Auswahl von ca. 1000 Haushalten beschränkten (vgl. Abb. 5). Eine entscheidende Rolle spielen dabei die räumliche Nähe zum Park, die Verfügbarkeit anderer Weideflächen, aber auch der physische Zugang, der z.B. von Westen durch steile Felsabstürze nahezu unmöglich ist.

\subsection{Entitlements: Wer nutzt den Khaptad Nationalpark?}

Doch wie kommt es, dass von den 10'000 Haushalten, die prinzipiell im Park Weidewirtschaft betreiben dürften, nur ca. 300 Haushalte dieses Verfügungsrecht (Entitlement) in Anspruch nehmen? Dem grössten Teil der Haushalte wird - wie bereits dargestellt durch traditionelle Institutionen bereits der Zugang (Ausstattung) verwehrt. Einigen Haushalten wurde die Nutzung der Weideressourcen verunmöglicht, weil ihre Ställe ausserhalb der offenen Weideflächen der Hochfläche lagen. Nach der Ausweisung des Parks mussten diese aufgegeben werden. Für die Erstellung neuer Ställe wurden die nötigen Genehmigungen von der Parkverwaltung nicht erteilt.

Die wichtigsten Institutionen, die die Umsetzung von der Ausstattung zu den Verfügungsrechten steuern, sind auf der Dorfebene im sozialen und ökonomischen Status der einzelnen Haushalte sowie deren Wirtschaftsstrategien zu suchen. So bedarf es, um die Ressource Sommerweide im Park sinnvoll zu nutzen, verschiedener Voraussetzungen: Es müssen genügend Tiere und Arbeitskraft vorhanden sein, diese zu betreuen, aber auch Bargeld, um die Gebühren zahlen zu können. Dies deutet zunächst darauf hin, dass es hauptsächlich grössere, wohlhabendere Haushalte sind, die diese Voraussetzungen erfüllen. Haushaltsbefragungen ergaben jedoch, dass auch viele ärmere Haushalte diese Ressource nutzen. Denn auch die von natürlichen Ressourcen unabhängigen Haushaltsstrategien, wie die Verfügbarkeit anderer Einkommensquellen spielen eine Rolle. Hier sind es besonders
Einkommen aus der in der Region weit verbreiteten temporären Arbeitsmigration nach Indien.

\subsection{Capabilities: Wer profitiert von der Nutzung der Ressourcen des Nationalparks?}

Tatsächlich nutzen insgesamt etwa nur 300 Haushalte aus acht Gemeinden die Weideressourcen des Nationalparks. Dabei handelt es sich um ca. $40 \%$ aller Haushalte aus wenigen Dörfern in der unmittelbaren Umgebung, die ihr Endowment zu Capablities umsetzen können. Doch wer profitiert davon?

Die Vorteile der Weidenutzung im Nationalpark liegen auf der Hand: Durch die Sömmerung auf den hochgelegenen Weideflächen bleiben die Tiere gesünder und geben mehr Milch. Der Druck auf die dorfnahen Weideressourcen und der Arbeitsaufwand sinken, da man weder Gras schneiden, noch die Tiere permanent überwachen muss. Beides führt dazu, dass man den Bestand an Tieren sogar erhöhen kann.

Innerhalb eines Haushaltes lassen sich Gewinner und Verlierer nicht so leicht ausmachen. Die sozial schlecht gestellten und stark überlasteten Frauen profitieren zwar durch die Entlastung beim Grasschneiden, müssen dafür aber die (fast immer) männliche Arbeitskraft ersetzen, die sich bei den Tieren auf der Hochfläche befindet.

\section{Schlussfolgerungen}

Am Beispiel des Tourismus im Gunung Mulu Nationalpark lässt sich zeigen, dass institutionelle Regelungen sich gleichzeitig ergänzen und verstärken aber auch widersprechen können (vgl. Abb. 4). Zwar kann der Schutz des Parks mit seinen Restriktionen als dominante Regelung gesehen werden, doch bleibt diese «heilige» Institution nicht unbeeinflusst von anderen institutionellen Regelungen, die von Touristen im Park berücksichtigt werden. Die Förderung von Tourismus im Nationalpark kann als Faktor gewertet werden, der die Idee, Regionen mit hoher Biodiversität oder besonderen Ökosystemen zu schützen, verstärkt und verbreitet. Die (westliche) Trennung von Natur und Kultur (vgl. Schiemann 1996) trägt dazu bei, die von Kultur (scheinbar) unberührte Natur als authentische Gegenwelt zum Alltag zu sehen, die es einerseits zu schützen gilt und die anderseits einen Anziehungspunkt für Reisende darstellt. Werden die Erwartungen der Reisenden im Park mehr oder weniger erfüllt (bzw. die Werte umgesetzt) - wie eine eigene Umfrage bei über 80 Reisenden in Mulu zeigt -, so wird die Regelung, dass Schutzgebiete ausgewiesen werden sollen, bestätigt. Und wird der Schutz nicht durch den Tourismus beeinträchtigt - wie es bei Überschreitungen von Tragfähigkeiten geschieht - so bestätigt sich die Regelung, dass es wünschbar ist, den Schutz von Gebieten mit touristischen Aktivitäten zu koppeln. Die so erfol- 
gende gegenseitige Verstärkung kann eine vergrößerte touristische Nachfrage nach Schutzgebieten zur Folge haben, was wiederum zur Ausweisung von neuen Schutzgebieten führen kann.

Decken sich jedoch die institutionellen Regelungen der Reisenden nicht so gut mit denen des Naturschutzes, wie sich dies durch die etwas anders gelagerten Bedürfnisse von (vor allem inländischen) Reisenden offenbart, so müssen die Widersprüche aufgelöst werden, will man nicht Gefahr laufen, dass Konflikte verstärkt auftreten. Dabei scheint sich die mächtige Institution «Naturschutz» durchzusetzen, die - gemäß der Bildungs- und Erziehungsfunktion von Parks Besucher aufklären und zur Einhaltung der bestehenden Regelungen im Park bewegen und somit «naturschutzkompatibler» machen soll.

Am Beispiel des Khaptad Nationalparks lässt sich verdeutlichen, dass es im Falle der Weidenutzung eher die oft als schwach bezeichneten, traditionellen, lokalen Institutionen sind, die die Inwertsetzung der Ressourcen entscheidend regeln. Wäre dies nicht der Fall, würde die Weideintensität stark zunehmen und die Ressourcen des Parks würden innerhalb kurzer Zeit übernutzt sein. Dies soll jedoch nicht darüber hinwegtäuschen, dass die neue Institution «Naturschutz» trotzdem einen großen Einfluss auf das alltägliche Leben der Menschen im Umkreis des Khaptad Nationalparks hat. Konflikte mit der Armee bei der Brennholzversorgung und zunehmende Ernteschäden durch die wachsende Zahl von Wildtieren belegen dies.

Die «heilige» Institution Naturschutz stellt dennoch nur für einen kleineren Teil der Bevölkerung momentan eine Einschränkung dar. Immer wieder kommen aber auch Überlegungen auf, die Weidenutzung des Parks vollkommen zu verbieten. Allein diese Überlegung zeigt, dass die Institution «Naturschutz» nicht nur wandelbar ist, sondern auch ein Sonderrecht beansprucht. Entscheidungen über Änderungen im Nutzungsreglement wurden bisher meist ohne Rücksprachen mit den Nutzern getroffen - einzig naturschutzrelevante Überlegungen spielten eine Rolle.

Die Verwendung des Environmental EntitlementsAnsatzes hat sich bei der Analyse von Problemen, die mit den institutionellen Regelungen von Ansprüchen an natürliche Ressourcen verbunden sind, bewährt. Egal, ob man die direkte Nutzung natürlicher Ressourcen durch die lokale Bevölkerung oder die eher der Erholung und Erbauung dienende touristische Nutzung durch nichtlokale Gruppen betrachtet, kann man mit ihm aufschlussreiche Ergebnisse erzielen. Die Rückkoppelung von Handlungen und den Rahmenbedingungen (hier Institutionen), aber auch die Machtausstattung verschiedener Interessensgruppen können gut herausgearbeitet werden. Am deutlichsten erscheinen die Vor-aber auch die Nachteile des Ansatzes bei der notwendigen Unterscheidung zwischen Endow- ments und Entitlements bzw. den Institutionen, die die Umsetzung beeinflussen. Sie sind konzeptionell klar ableitbar, was die analytische Tiefe verbessert, doch lassen sie sich am Einzelbeispiel oft nur schwer differenzieren. Daneben kann nicht nur die Nutzung spezifischer Ressourcen offen gelegt werden, sondern es werden - durch die Benennung der einschränkenden Institutionen - auch die ansonsten oft übergangenen «Nichtnutzer» sichtbar.

Die beiden Beispiele belegen, dass die institutionellen Regelungen des internationalen Naturschutzes durchaus eine gewisse «Heiligkeit» beanspruchen, indem sie sich als mächtigster Anspruch meist durchsetzen, vor allem dann, wenn Veränderungen als Beeinträchtigung des Ökosystems oder der Artenvielfalt wahrgenommen werden. Allerdings sind die Regelungen oft nicht so rigid, wie man es sich vorstellen könnte und können dem Kontext des Parks angepasst werden. Was oft auch wenig beachtet wird, sind die unterschiedlichen zeitlichen Horizonte der von den Regelungen Betroffenen. Während in Khaptad die lokale Bevölkerung in ihrem täglichen Lebenszusammenhang sehr stark von Veränderungen der Institutionen im Park betroffen ist, spielt dies für die einzelnen Touristen, die Mulu besuchen wollen, eine weit geringere Rolle, da es sie lediglich einige Tage betrifft und sie gut ausweichen könnten. Allerdings hat die touristische Nutzung als Ganzes betrachtet einen großen Einfluss auf die am und im Park lebende Bevölkerung sowie auf die Tourismusindustrie der Region.

\section{Literatur}

Backhaus, N. (1999): Rummel im Dschungel. - In: Mosquito - Die entwicklungspolitische Zeitschrift der Schweiz 1999/1: 21-26.

Backhaus, N. (2000): The Traveller's Gaze - Ecotourism in National Parks of Malaysian Borneo. - 6th Biennal Borneo Research Conference Vol. 4, Kuching/ Sarawak: Institute of East Asian Studies: 440-450.

Bauman, Z. (1997): Postmodernity and its Discontents. - New York: New York University Press.

Bernbaum, E. (1996): Sacred Mountains - Implications for Protected Area Management. - In: PARKS 6/1: 41-48.

Blaikie, P. \& S.J. Jeanrenaud (1997): Biodiversity and Human Welfare. - In: Ghimire, K.B. \& M.P. Pimbert (Hrsg.): Social Change and Conservation. - London: Earthscan: 46-70.

BorRini-Feyerabend, G. (Hrsg.) (1997): Beyond Fences - Seeking Social Sustainability in Conservation. Gland: IUCN.

Colchester, M. (1997): Salvaging Nature: Indigenous Peoples and Protected Areas. - In: Ghimire, K.B. \& M.P. Pimbert (Hrsg.): Social Change and Conservation. - London: Earthscan: 100-130.

Cubitt, G. \& J. Payne (1996): Wild Malaysia - The 
Wildlife and Scenery of Peninsular Malaysia, Sarawak and Sabah. - Singapore: New Holland.

ElKana, Y. (1986): Anthropologie der Erkenntnis Die Entwicklung des Wissens als episches Theater einer listigen Vernunft. - Frankfurt am Main: Suhrkamp.

Ghimire, K.B. \& M.P. Pimbert (1997): Social Change and Conservation: An Overview of Issues and Concepts. - In: Ghimire, K.B. \&. M.P. Pimbert (Hrsg.): Social Change and Conservation. - London: Earthscan: $1-45$.

GidDENS, A. (1995): Die Konstitution der Gesellschaft - Grundzüge einer Theorie der Strukturierung. - = Theorie und Gesellschaft 1, Frankfurt am Main, New York: Campus-Verlag.

Harrisson, T. (1996): Kinabalu, The Wonderful Mountain of Change. - In: Wong, K.M. \& A. Phillipps (Hrsg.): Kinabalu - Summit of Borneo. - Kota Kinabalu: The Sabah Society: 1-18.

Hutron, W. (1998): Sarawak. - Singapore: APA Publications (Insight Pocket Guides).

INSKIPP, C. (1989): The Ornithological Importance of Khaptad Nationalpark, Nepal. - In: Forktail 5: 49-60.

IUCN, UNEP \& WWF (1991): Caring for the Earth A Strategy for Sustainable Living. - Gland.

IUCN (1993): Nature Reserves of the Himalaya and the Mountains of Central Asia. - New Delhi, IUCN.

IUCN (1994): Guidelines for Protected Area Management Categories. - Gland: IUCN.

IUCN (1998): 1997 United Nations List of Protected Areas. - Gland: IUCN.

Kollmair, M. \& U. MÜller-BöKer (2001): Environmental Entitlements: A New Approach to Analyse Park-People Interactions - The Example of Khaptad National Park. - International Symposium on the Himalayan Environments: Mountain Sciences and Ecotourism/Biodiversity, Kathmandu, 24.-26. November 2000 (in press).

KüPFER, I. (2000): Die regionalwirtschaftliche Bedeutung des Nationalparktourismus untersucht am Beispiel des Schweizerischen Nationalparks. $-=$ Nationalpark-Forschung in der Schweiz 90, Zernez.

Leach, M., Mearns, R. \& I. Scoones (1999): Environmental Entitlements - Dynamics and Institutions in Community-Based Natural Resource Management. In: World Development 27/2: 225-247.

LuHMANN, N. (1988): Njet-Set und Terror-Desperados. - In: Gente, P., Paris, H. \& M. Weinmann (Hrsg.): Niklas Luhmann - Short Cuts. - Frankfurt am Main: Zweitausendeins: 64-74.

MülLER-BöKER, U. (2000): Ecotourism in Nepal: The Example of the Royal Chitawan National Park. - In: ThaPA, R.P. \& J. BAADEN (eds): Nepal: Myths and Realities, Delhi: 100-117.

MÜller-BöKER, U. \& M. Kollmair (2000): Livelihood Strategies and Local Perceptions of a New Nature Conservation Project in Nepal. The Kanchenjunga
Conservation Area Project. - In: Mountain Research and Development 20/4: 324-331.

NESAC, Nepal South Asian Centre (1998): Nepal Human Development Report 1998. - Kathmandu.

NorTH, D.C. (1990): Institutions, Institutional Change and Economic Performance. - Cambridge, Melbourne: Cambridge University Press.

Querol, D. (1992): Genetic Resources, Our Forgotten Treasure - Technical and Socio-Economic Approaches. - Penang: Third World Network.

SarawaK Tourism Board (1997): Sarawak - The Hidden Paradise of Borneo. - Kuching/Sarawak (Video).

Schiemann, G. (Hrsg.) (1996): Was ist Natur? - Klassische Texte zur Naturphilosophie. - München: Deutscher Taschenbuch Verlag,

Sen, A. (1981): Poverty and Famines - An Essay on Entitlement and Deprivation. - Oxford: Clarendon Press.

Thambiah, S. (2000): Collective Representation and Individual Experience - The Bhuket of Sarawak and the Individuation of their Religion. - 6th Biennal Borneo Research Conference Vol.1, Kuching/Sarawak: Institute of East Asian Studies.

UNESCO (2000): World Heritage Committee Inscribes 61 New Sites on World Heritage List. - UNESCO, http://www.unesco.org/whc/nwhc/pages/news/main2.htm (accessed 14.12.2000).

URRY, J. (1990): The Tourist Gaze - Leisure and Travel in Contemporary Societies. - London: Sage.

WiLson, E.O. \& F.M. Peter (1988): Biodiversity. - Washington, DC: National Academy Press.

WWF (2000): Living Planet Campaign. - WWF, http:// panda.org/livingplanet (accessed 20.12.2000).

\section{Zusammenfassung: Heilige Institutionen? - Regelungen von Nutzungsansprüchen an Ressourcen von Nationalparks}

Naturschutzgebiete bergen eine Vielzahl von Ressourcen, deren Nutzung von verschiedenen Akteuren angestrebt wird. In diesen «Handlungsarenen» laufen nahezu idealtypisch Prozesse ab, die durch ein breites Spektrum institutioneller Regelungen beeinflusst werden, die zwischen lokaler und internationaler Ebene angesiedelt sind. Eines scheint Konflikte um institutionelle Regelungen in und um Schutzgebiete grundsätzlich zu kennzeichnen: Der wie auch immer geartete Schutz von «Natur» wird als Zielvorstellung immer allen anderen Zielen übergeordnet. Der Naturschutz kann somit zu einer unantastbaren «heiligen Institution» werden, die nicht in Frage gestellt werden darf. Die in einem Schutzgebiet gesehenen Werte kann man in «direkte», «indirekte» und "optionale» Nutzwerte unterteilen. Die Perzeption und Gewichtung dieser Werte ist bei den beteiligten Akteursgruppen oft recht verschieden.

Anhand zweier Beispiele aus Malaysia und Nepal 
kann gezeigt werden, dass die institutionellen Regelungen des internationalen Naturschutzes durchaus eine gewisse «Heiligkeit» beanspruchen, indem sie sich als mächtigster Anspruch meist durchsetzen, auch wenn sie dem spezifischen Kontext eines Parks angepasst werden.

\section{Summary: Sacred Institutions? - Régulation of land- use entitlement in national parks}

Nature conservation areas offer a wide range of landuse possibilities which various actors are keen to exploit. The decision processes related to these protected areas are influenced by local and international expectations, as well as by a broad range of institutional regulations. The variety of regulation forms have a common base: the protection of «nature» has priority over all other goals. Thus, there is the danger that nature protection could develop into a sacrosanct «sacred institution», which one may not question. The values that are seen in a conservation area by different actors can be differentiated between «direct», «indirect», and "optional» use values. The perception and estimation of these values can differ considerably. Based on two examples from Malaysia and Nepal we can show that the institutional regulation of international conservation of nature indeed claims a certain sacredness. Even if it is adapted to the specific context of a given park, nature conservation per se has the upper hand.

\section{Résumé: Des institutions saintes? - Réglementations des revendications d'exploitation des ressources des parcs nationaux}

Les réserves naturelles renferment une multitude de ressources que différents acteurs cherchent à exploiter. Influencés par un vaste éventail de réglementations institutionnelles situées entre un niveau local et un niveau international, des processus types caractéristiques se déroulent dans ces «arènes». Un point commun semble caractériser les conflits pour les réglementations institutionnelles à l'intérieur et autour des réserves naturelles: la protection de la «nature» est toujours placée au-dessus de tous les autres objectifs.
Ainsi, la protection de la nature peut devenir une «institution sainte» inviolable qui ne peut être remise en question. On peut subdiviser les valeurs perçues dans une réserve naturelle par les divers acteurs en valeurs d'usage «directes», «indirectes» et «optionnelles». La perception et la pondération de ces valeurs peuvent varier considérablement selon les groupes d'acteurs concernés.

L'exemple de deux parcs naturels situés en Malaisie et au Népal montre que les réglementations institutionnelles de la protection internationale de la nature, en s'imposant le plus souvent comme la revendication la plus puissante, doivent s'appuyer sur une certaine «sainteté», même si elles sont adaptées au contexte spécifique d'un parc donné.

Dr. Norman Backhaus, Dr. Michael Kollmair, Geographisches Institut der Universität Zürich, Abteilung Anthropogeographie, Winterthurerstrasse 190, CH-8057 Zürich.

e-mail:

backhaus@geo.unizh.ch

kollmair@geo.unizh.ch

\section{Manuskripteingang/received/rentrée du manuscrit:} 14.2.2001

Annahme zum Druck/accepted for publication/ acceptation à l'impression: 17.4.2001 